\title{
Observation of coherent transition radiation in the prewave zone
}

\author{
Changmook Yim (임창묵), ${ }^{1}$ Junho Ko (고준호), ${ }^{1}$ Seonghoon Jung (정성훈), ${ }^{2}$ Daehoon Han (한대훈), ${ }^{3}$ \\ Changbum Kim (김창범), ${ }^{2}$ Jaehun Park (박재헌), ${ }^{2, *}$ Heung-Sik Kang (강흥식), ${ }^{2, \dagger}$ and In Soo Ko (고인수) ${ }^{1}$ \\ ${ }^{1}$ Department of Physics, POSTECH, Pohang 790-784, South Korea \\ ${ }^{2}$ Pohang Accelerator Laboratory, POSTECH, Pohang 790-784, South Korea \\ ${ }^{3}$ Department of Physics, KAIST, Daejeon 305-701, South Korea
}

(Received 30 December 2011; published 19 March 2012)

\begin{abstract}
Transition radiation generated from a relativistic electron beam is used as a tool for diagnostics of the beam. Also, it can be used as a source of femtosecond (fs) THz radiation. We are generating the THz radiation with the pulse energy of $3 \mu \mathrm{J}$ by means of the coherent transition radiation (CTR) from 55-MeV electron beam in the fs-THz linac at the Pohang Accelerator Laboratory. The spatial distribution of CTR could be easily observed by using a pyroelectric camera. In this paper, we report observation of the transversal distribution of CTR in the prewave zone, and compare with calculated results based on the bunch form factor.
\end{abstract}

DOI: 10.1103/PhysRevSTAB.15.030706

PACS numbers: 41.60.Dk, 41.75.Ht

\section{INTRODUCTION}

A fs- $\mathrm{THz}$ linac was constructed to generate femtosecond $\mathrm{THz}$ radiation by using an ultrashort electron beam at the Pohang Accelerator Laboratory (PAL) [1,2]. A photocathode rf gun and a chicane bunch compressor were taken up for the generation of a fs electron beam in the linac. Now, the electron beam with the 55-MeV energy, the 200$\mathrm{pC}$ charge, and the 350-fs bunch length is generated at the $10-\mathrm{Hz}$ repetition rate in the linac, and the $3-\mu \mathrm{J} \mathrm{THz}$ pulse is radiated from the electron beam through transition radiation $[1,2]$.

Coherent radiation is emitted from the electron bunch whose length is shorter than the radiation wavelength. The total spectral radiation power from a monoenergetic bunch of $N$ electrons is [3]

$$
P(\omega)=p(\omega) N[1+(N-1) f(\omega)],
$$

where $p(\omega)$ is the spectral radiation power from single electron, and $f(\omega)$ is the bunch form factor, which is the Fourier transform of the normalized bunch distribution $[F(\vec{r})]$ squared, $f(\omega)=\left|\int e^{i \vec{k} \cdot \vec{r}} F(\vec{r}) d^{3} r\right|^{2}$, where $\vec{r}$ is the electron position from the bunch center; longitudinal bunch form factor $f(\omega)=\exp \left(-\omega^{2} \sigma^{2} / c^{2}\right)$ for a Gaussian distribution with the standard deviation of $\sigma$ and $f(\omega)=[\sin (\omega l / 2 c) /(\omega l / 2 c)]^{2}$ for a rectangular distribution with a width of $l$. The first term and the second term in the square bracket correspond to incoherent radiation and coherent radiation, respectively. When the form

\footnotetext{
*jaehunpa@postech.ac.kr

†hskang@postech.ac.kr
}

Published by the American Physical Society under the terms of the Creative Commons Attribution 3.0 License. Further distribution of this work must maintain attribution to the author(s) and the published article's title, journal citation, and DOI. factor is close to 1 , the coherent radiation has the $N$ times higher power than the incoherent radiation.

Transition radiation is emitted when a charged particle crosses the boundary between two media with different dielectric constants. It was first predicted by Ginzburg and Frank [4], and since that time the characteristics of transition radiation have been extensively studied [5-11].

Transition radiation in the $\mathrm{THz}$ regime becomes coherent when it is generated from the electron bunch shorter than the $\mathrm{THz}$ wavelength. Transition radiation has been used for beam diagnostics, and especially coherent transition radiation (CTR) gives a great tool of diagnosing the bunch length of subpicosecond electron beam [12-17].

The observation results for CTR have been presented in other literature $[6,7,10,14]$. However, the conventional methods for CTR observation using Golay cell detector, pyroelectric detector, etc. have drawbacks to be difficult and complicated because they are not a single-frame method. A pyroelectric array camera [Pyrocam III, Spiricon, Inc. (A spectral response range of Pyrocam III is 13-355 and $1.06-3000 \mu \mathrm{m}$, and a sensitivity is $7 \mathrm{~nJ}$ per pixel.)] gives the way that CTR observation can be easily fulfilled because it offers discernible results for the transversal distribution of CTR. In this paper, we experimentally confirm the theoretical results using the pyroelectric camera.

The remainder of the paper is organized as follows. In the second section, we show the observation results of CTR. In the third section, the observation results are compared with the theoretical results using the transverse beam distribution generated at the PAL fs- $\mathrm{THz}$ linac and the bunch form factor obtained from a streak camera image. Finally, the paper concludes in the fourth section.

\section{CTR OBSERVATION RESULTS}

The CTR target in the PAL fs-THz linac is equipped with a titanium (Ti) foil whose radius and thickness are 0.5 


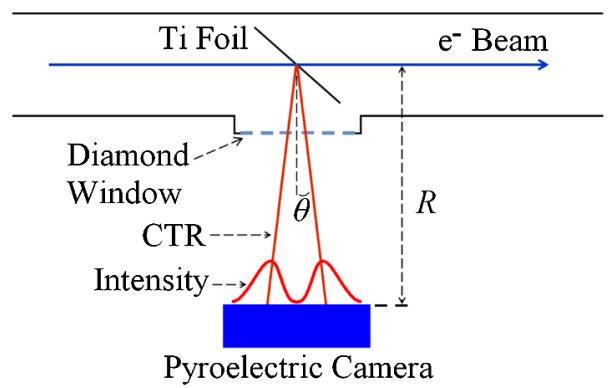

FIG. 1. Setup diagram for the spatial distribution measurement of CTR.

inch and $1 \mu \mathrm{m}$, respectively. The experimental setup for CTR observation is shown in Fig. 1. After CTR emitted from $\mathrm{Ti}$ foil passes through the diamond window, its spatial profile is directly taken by a Pyrocam III located at the position of $R$ from the CTR target.

Figure 2 shows the spatial distribution of the CTR at the position of $R=11.0 \mathrm{~cm}$. Figure 2(a) shows a single frame of the CTR image, but the image is indistinct because the CTR intensity is lower than the detection limit of the Pyrocam III. Figure 2(b) shows the $\mathrm{THz}$ beam profile which is a sum of 100 frames, and Fig. 2(c) is the cross section along the solid arrow in Fig. 2(b).

The significant characteristics of the transition radiation in Fig. 2 exist in a complicated azimuthal asymmetry and a peak-intensity angle. The angle of the maximum intensity is $28.2 \pm 2.7 \mathrm{mrad}$. In the next section, the azimuthal asymmetry and the observed maximum-intensity angle will be discussed.
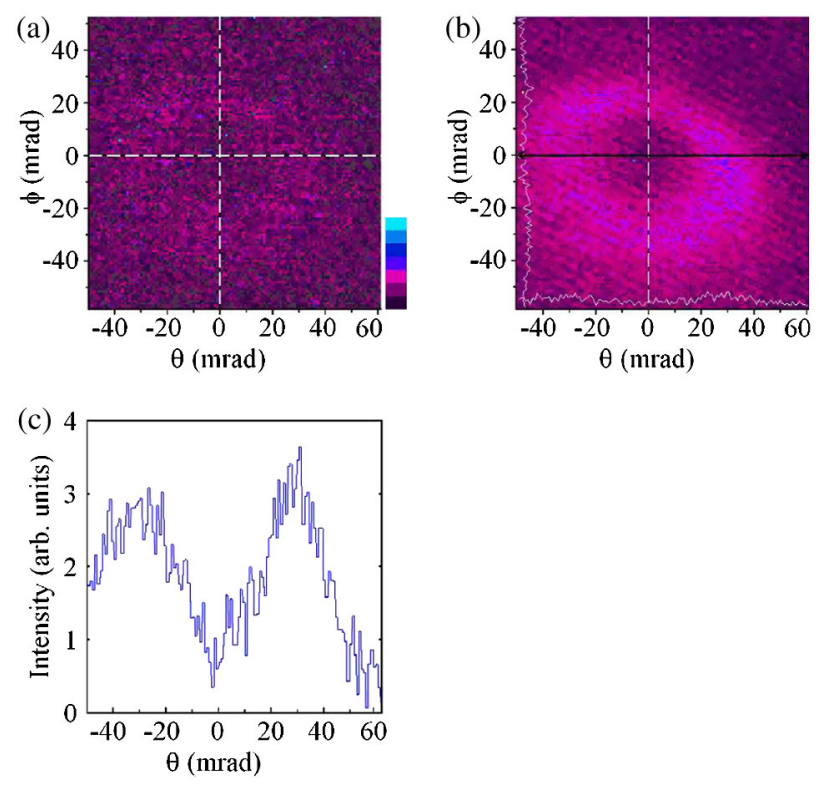

FIG. 2. (a) Spatial distribution of CTR at $R=11.0 \mathrm{~cm}$, (b) CTR image summed up to 100 frames, and (c) CTR intensity along the solid arrow in (b).

\section{OBSERVATION RESULTS ANALYSIS}

\section{A. Azimuthal asymmetry}

A CTR radiator, generally, uses a target rotated by $\pi / 4$ into the electron beam path because of its structural simplification. The intensity distribution of the transition radiation from a $\pi / 4$ oblique target has a simple azimuthal asymmetry [10,18]. However, Fig. 2 shows a complicated azimuthal distribution. We supposed that the complication comes from the electron beam with a nonuniform transverse distribution, and the equations were derived using Casalbuoni's paper [19] to confirm the effect of the transverse beam distribution on the spatial distribution of CTR [20]:

$$
\frac{d^{2} U}{d \omega d \Omega} \propto R^{2}\left(\left|E_{x}\right|^{2}+\left|E_{y}\right|^{2}\right)
$$

with

$$
\begin{aligned}
E_{x, y} \propto & \frac{k^{2}}{\beta^{2} \gamma} \frac{\exp (i k R)}{R} \int_{0}^{a} \int_{0}^{2 \pi} \int_{B} K_{1}\left(\frac{k \rho^{\prime}}{\beta \gamma}\right) \\
& \times\left\{\begin{array}{l}
\cos \varphi^{\prime} \\
\sin \varphi^{\prime}
\end{array}\right\} f\left(s, \varphi_{s}\right) \exp \left(\frac{i k \rho^{2}}{2 R}\right) \\
& \times \exp [-i k \rho(\sin \theta \cos \phi \cos \varphi \\
& +\cos \theta \sin \phi \sin \varphi)] \rho d B d \varphi d \rho
\end{aligned}
$$

and

$$
\begin{aligned}
& \rho^{\prime}=\sqrt{\rho^{2}+s^{2}-2 \rho s \cos \left(\varphi-\varphi_{s}\right)}, \\
& \varphi^{\prime}=\arg \left[\left(\rho \cos \varphi-s \cos \varphi_{s}\right)+i\left(\rho \sin \varphi-s \sin \varphi_{s}\right)\right],
\end{aligned}
$$

where $K$ is the modified Bessel function, $k$ is wave number, $a$ is target radius, $\theta$ and $\phi$ are the projected angles taken in the $x$-axis and the $y$-axis directions, respectively, and $B$ and $f\left(s, \varphi_{s}\right)$ are the area and the transverse density function of the electron beam, respectively. Figure 3 shows the numerical result of Eq. (2). The image, whose size is $2.8 \mathrm{~mm} \times 2.7 \mathrm{~mm}$, at the top right corner is the beam distribution, which was generated at the PAL fs-THz linac, used in the numerical calculation. The azimuthal asymmetry in Fig. 3 is more complicated than the asymmetry [11,18] of the distribution for $\pi / 4$ incidence transition radiation from an electron. Therefore, we can ascertain that the complicated azimuthal distribution of the observation results in Fig. 2 is due to the effect of the transverse beam distribution.

Figure 4 shows $\theta$ and $\phi$ cross sections of distribution in Fig. 3. In the figure, the intensity distributions of transition radiation from single electron and the uniform electron beam with radius of $1.2 \mathrm{~mm}$, which is similar to the beam size in Fig. 3, are shown, too. The deviation of peak-intensity angle due to the nonuniformity of the transverse beam distribution is bigger than the one coming from 

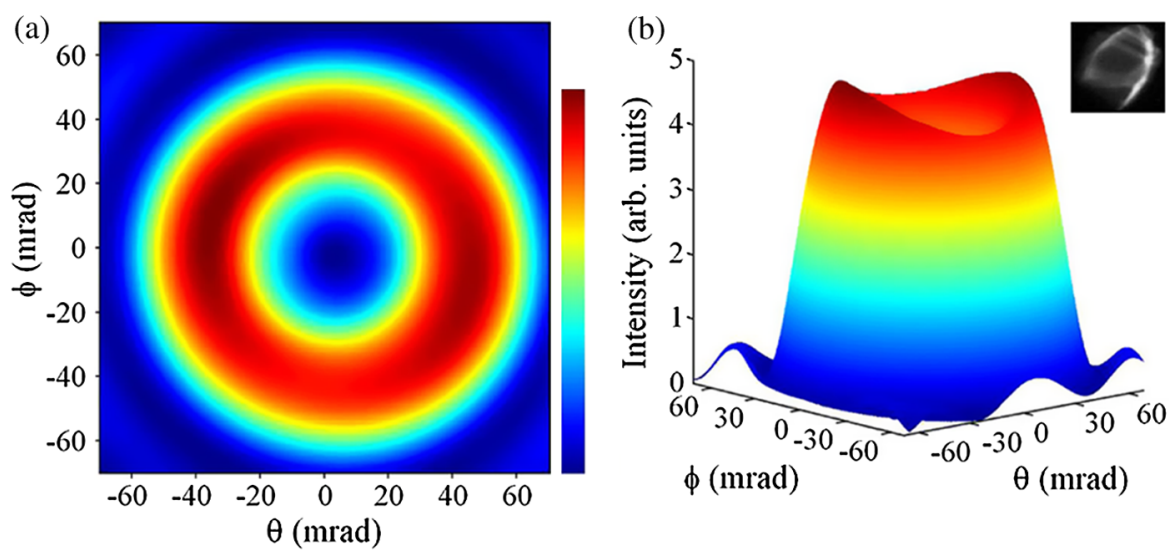

FIG. 3. (a) 2D and (b) 3D plot for the spatial distribution of transition radiation from the electron beam with a transverse distribution at $R=0.1 \mathrm{~m}$. The radiation frequency is $1 \mathrm{THz}$, and the beam image used in the numerical calculation is at the top right corner.

the uniform electron beam with a finite size. We do not take into account the effect of transverse beam distribution on the peak-intensity angle of calculation results. The deviation of peak-intensity angle is just considered as the experimental errors.

\section{B. Peak-intensity angle}

For comparison with the experimental results, we calculated the total intensity of transition radiation in the prewave zone. The spectral-angular radiation density for $\pi / 4$ incidence transition radiation in the prewave zone can be obtained by the equations that Karlovets suggested [11]. For a circular target case, the equations are slightly modified, and we only take into account the case of $\phi=0$ :

$$
\frac{d^{2} U}{d \omega d \Omega}=c R^{2}\left(\left|E_{x}\right|^{2}+\left|E_{y}\right|^{2}\right)
$$

with

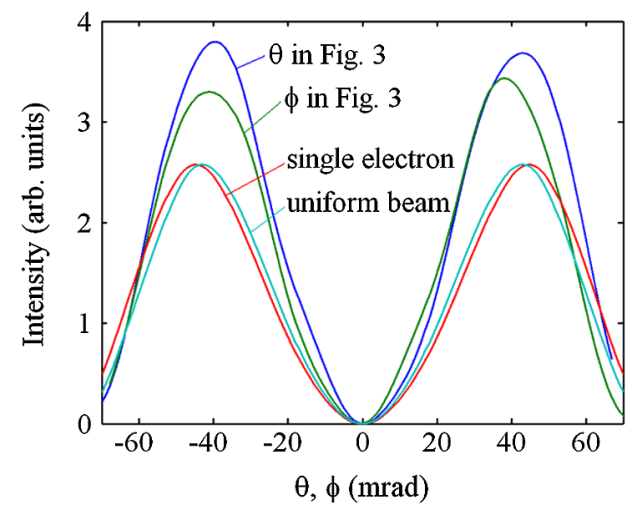

FIG. 4. $\theta$ and $\phi$ cross sections of distribution in Fig. 3, intensity distribution of transition radiation from single electron, and intensity distribution of transition radiation from the uniform electron beam with radius of $1.2 \mathrm{~mm}$, which is similar to the beam size in Fig. 3.

$$
\begin{aligned}
E_{x}= & \frac{2 i e}{c \beta^{2} \gamma \lambda^{2}} \frac{\cos (\theta+\pi / 4)}{R} \\
& \times \int_{0}^{a} \int_{0}^{2 \pi} \frac{r^{2} \cos \vartheta}{p} K_{1}\left(\frac{2 \pi}{\beta \gamma \lambda} p\right) e^{i \varphi} d \vartheta d r, \\
E_{y}= & \frac{2 i e}{c \beta^{2} \gamma \lambda^{2}} \frac{1}{R} \int_{0}^{a} \int_{0}^{2 \pi}\left[\frac{0.5 r \sin \vartheta}{p} K_{1}\left(\frac{2 \pi}{\beta \gamma \lambda} p\right)\right. \\
& \left.-\frac{i}{\sqrt{2} \gamma} K_{0}\left(\frac{2 \pi}{\beta \gamma \lambda} p\right)\right] r e^{i \varphi} d \vartheta d r,
\end{aligned}
$$
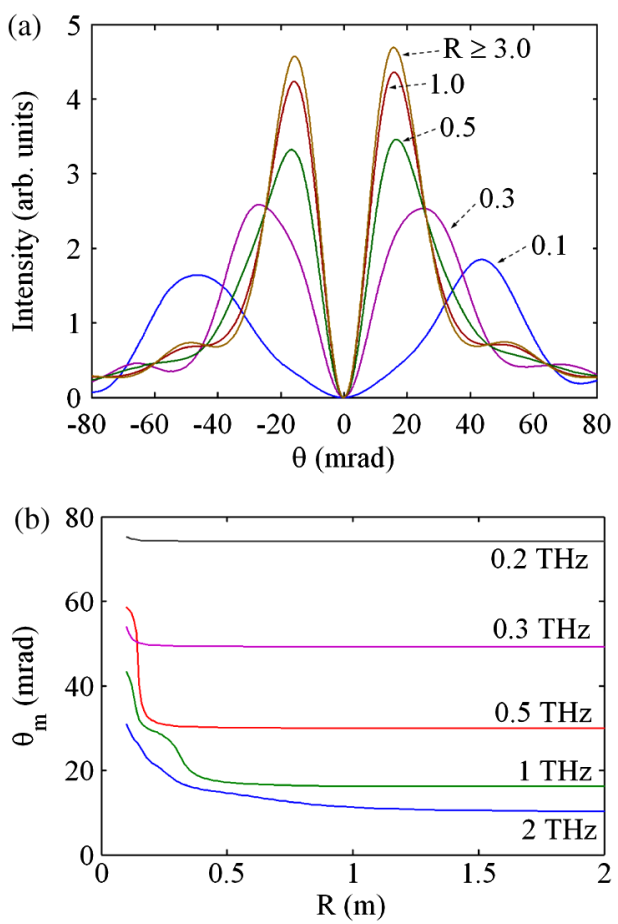

FIG. 5. (a) Transition radiation intensity with 1-THz frequency for different distances from the target and (b) the peak-intensity angles $\theta_{m}$ as a function of the distance from the target for different frequencies. Numbers indicating the curves in (a) are distances $R$ in units of $\mathrm{m}$. 
and

$$
\begin{aligned}
p= & \sqrt{r^{2} \cos ^{2} \vartheta+0.5 r^{2} \sin ^{2} \vartheta}, \\
\varphi= & k\left(R-r \sin \vartheta\left[\sin \left(\theta+\frac{\pi}{4}\right)-\frac{1}{\sqrt{2} \beta}\right]\right. \\
& \left.+\frac{1}{2 R}\left\{r^{2} \cos ^{2} \vartheta+r^{2} \sin ^{2} \vartheta\left[1-\sin ^{2}\left(\theta+\frac{\pi}{4}\right)\right]\right\}\right) .
\end{aligned}
$$

Figure 5(a) shows the transition radiation intensity at various distances from the CTR target for the frequency of $1 \mathrm{THz}$. The two lobes of the distributions become wide at a close position to the CTR target. In Fig. 5(b), the peak angles $\theta_{m}$ for different frequencies are given as a function of the distance from the target. It is shown that the peakintensity angles in each frequency become constant at distant $R$, i.e., in the far field [8].

The total spatial intensity of the transition radiation can be written by

$$
\frac{d U}{d \Omega} \propto \int f(\omega)\left(\frac{d^{2} U}{d \omega d \Omega}\right) d \omega,
$$

where $f(\omega)$ is the longitudinal bunch form factor. The form factor is calculated using the bunch distribution obtained from a streak camera (FESCA-200, Hamamatsu Photonics
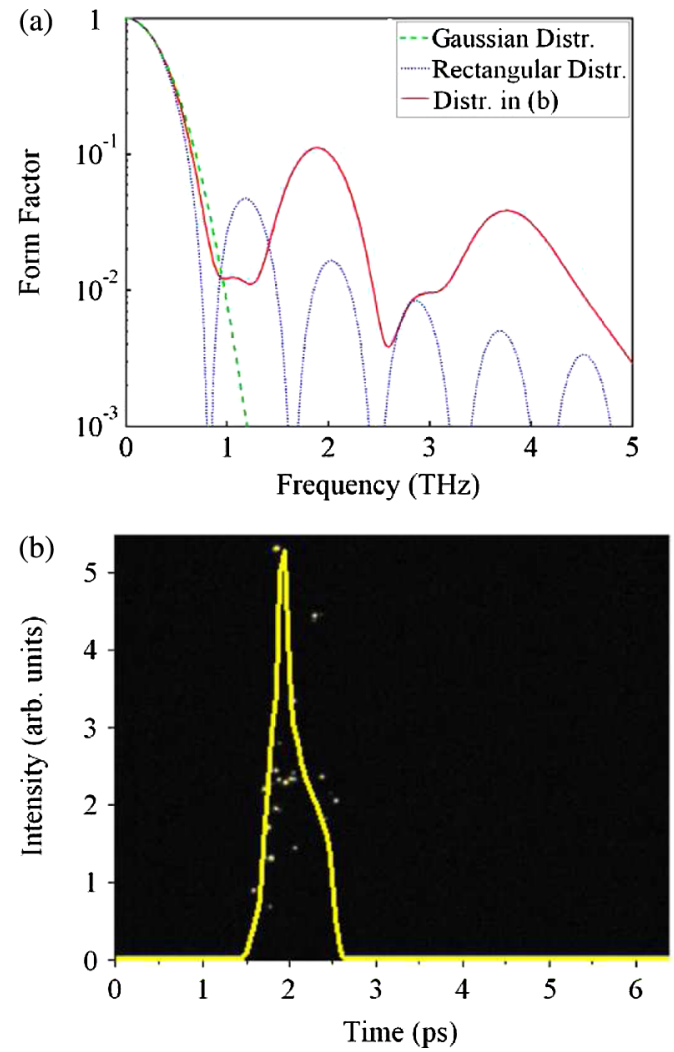

FIG. 6. (a) Bunch form factors for the Gaussian distribution, the rectangular distribution, and the bunch distribution in (b), and (b) streak camera image (bright points) and longitudinal bunch distribution (solid line) obtained from that.

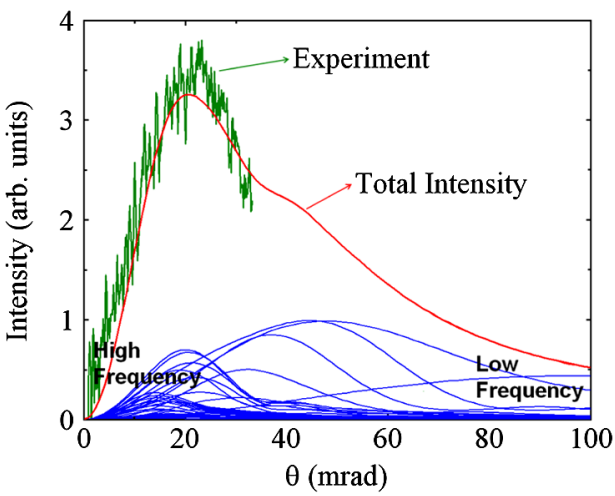

FIG. 7. Spatial intensity distributions for different frequencies, total spatial intensity, and experimental result at $R=25 \mathrm{~cm}$.

K.K.). Figure 6(a) shows the form factors for the Gaussian distribution, the rectangular distribution, and the bunch distribution measured by the streak camera, which is shown in Fig. 6(b). In Fig. 6(a), the rms lengths of the three distributions are all $350 \mathrm{fs}$. The total spatial intensity has a lower and an upper limit about the frequency by the spatial intensity $d^{2} U / d \omega d \Omega$ and the bunch form factor $f(\omega)$, respectively.

Figure 7 shows the spatial intensity distributions for different frequencies and the total spatial intensity of the transition radiation at $R=25 \mathrm{~cm}$. The radiation intensity is weaker at high frequencies and low frequencies compared to frequencies in between. The spatial intensity of the experimental result is also shown in Fig. 7. The peakintensity angle of the experimental result is in agreement with the one of the calculated result at the angle of near $22 \mathrm{mrad}$. Figure 8 shows the peak-intensity angle as a function of distance from the target. The plot marked with "Calculation1" in Fig. 8 is calculated by using a

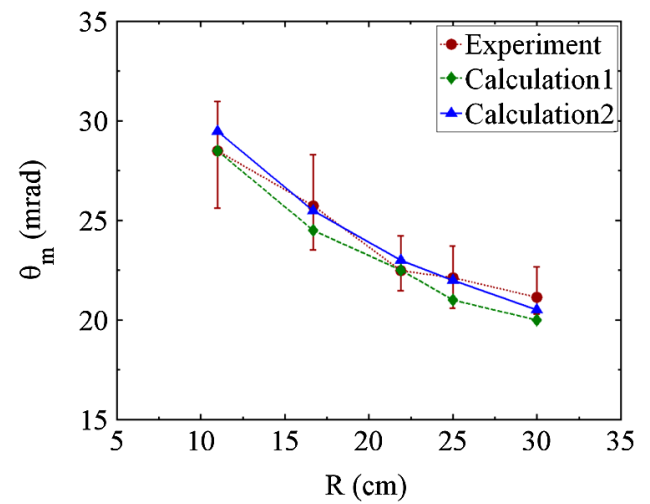

FIG. 8. Peak-intensity angles of CTR distributions for various distances. Calculation1 is obtained from a bunch form factor of the bunch distribution in Fig. 6(b), and Calculation2 is from the summation of the spatial intensities calculated by using the bunch form factors for the bunch distributions measured by the streak camera. Note that the spatial CTR image was summed up to 100 frames. 
bunch form factor of the bunch distribution in Fig. 6(b), and the plot marked with "Calculation2" is obtained from the summation of the spatial intensities calculated by using the bunch form factors for the bunch distributions measured by the streak camera.

From the calculation of the peak-intensity angle using the bunch form factors of the electron bunch generated in the fs- $\mathrm{THz}$ linac, we ascertained that the angle agrees with the one found from the CTR image taken by a pyroelectric camera. Inversely, if the electron bunches have a similar distribution regardless of their length, and a single-frame CTR image is obtained by using a pyroelectric camera, the bunch length can be estimated from the peak-intensity angle of the CTR image and Eq. (4).

\section{CONCLUSION}

We have shown the theoretical results and the experimental results for the spatial distribution of CTR. The theoretical results were calculated by using the bunch form factor of the bunch distribution obtained from a streak camera, and compared with the experimental results measured by a pyroelectric camera.

The observation results of CTR are affected by a water absorption [21] of $\mathrm{THz}$ radiation, a diamond vacuum window $[19,22]$, etc. as well as a transverse distribution of an electron beam. In spite of these factors, we ascertained that the calculations and experiments for the peak-intensity angles of spatial CTR distribution in the prewave zone accord with each other.

Finally, if enough radiation energy to measure a singleframe image can be reached, it might be possible to derive the shot to shot electron bunch length from the peakintensity angle of the spatial CTR distribution.

\section{ACKNOWLEDGMENTS}

This work was supported by the National Research Foundation of Korea (NRF) funded by the Ministry of Education, Science and Technology (MEST) (No. 20080059842, No. 2010-0012016, and No. 2011-0001291).

[1] J. Park, C. Kim, J. Lee, C. Yim, C. H. Kim, J. Lee, S. Jung, J. Ryu, H. S. Kang, and T. Joo, Rev. Sci. Instrum. 82, 013305 (2011).
[2] C. Yim, J. Hong, Y. Parc, S. Jung, D. Han, J. Ryu, J. Park, H. S. Kang, and I. S. Ko, Rev. Sci. Instrum. 82, 106104 (2011).

[3] C. J. Hirschmugl, M. Sagurton, and G. P. Williams, Phys. Rev. A 44, 1316 (1991).

[4] I. M. Frank and V.L. Ginzburg, J. Phys. USSR 9, 353 (1945).

[5] V. L. Ginzburg, Phys. Scr. T2/1, 182 (1982).

[6] U. Happek, A. J. Sievers, and E. B. Blum, Phys. Rev. Lett. 67, 2962 (1991).

[7] Y. Shibata, K. Ishi, T. Takahashi, T. Kanai, F. Arai, S. I. Kimura, T. Ohsaka, M. Ikezawa, Y. Kondo, R. Kato, S. Urasawa, T. Nakazato, S. Niwano, M. Yoshioka, and M. Oyamada, Phys. Rev. E 49, 785 (1994).

[8] V. A. Verzilov, Phys. Lett. A 273, 135 (2000).

[9] S. N. Dobrovolsky and N. F. Shul'ga, Phys. At. Nucl. 64, 994 (2001).

[10] D. Sütterlin, D. Erni, M. Dehler, H. Jäckel, H. Sigg, and V. Schlott, Nucl. Instrum. Methods Phys. Res., Sect. B 264, 361 (2007).

[11] D. V. Karlovets and A. P. Potylitsyn, Nucl. Instrum. Methods Phys. Res., Sect. B 266, 3738 (2008).

[12] C. T. Settakorn, Coherent $\mathrm{THz}$ Transition Radiation: Generation, Characterization and Applications (VDM Verlag, Saarbrücken, 2009).

[13] D. W. Rule, Nucl. Instrum. Methods Phys. Res., Sect. B 24/25, 901 (1987).

[14] Y. Shibata, T. Takahashi, T. Kanai, K. Ishi, M. Ikezawa, J. Ohkuma, S. Okuda, and T. Okada, Phys. Rev. E 50, 1479 (1994).

[15] H. C. Lihn, P. Kung, C. Settakorn, H. Wiedemann, and D. Bocek, Phys. Rev. E 53, 6413 (1996).

[16] A. Murokh, J. B. Rosenzweig, M. Hogan, H. Suk, G. Travish, and U. Happek, Nucl. Instrum. Methods Phys. Res., Sect. A 410, 452 (1998).

[17] T. Watanabe, M. Uesaka, J. Sugahara, T. Ueda, K. Yoshii, Y. Shibata, F. Sakai, S. Kondo, M. Kando, H. Kotaki, and K. Nakajima, Nucl. Instrum. Methods Phys. Res., Sect. A 437, 1 (1999).

[18] C. Settakorn, Ph.D. thesis, Stanford University, Stanford, 2001.

[19] S. Casalbuoni, B. Schmidt, P. Schmüser, V. Arsov, and S. Wesch, Phys. Rev. ST Accel. Beams 12, 030705 (2009).

[20] C. Yim, Ph.D. thesis, POSTECH, Pohang, 2012.

[21] X. Xin, H. Altan, A. Saint, D. Matten, and R. R. Alfano, J. Appl. Phys. 100, 094905 (2006).

[22] V. V. Kubarev, Nucl. Instrum. Methods Phys. Res., Sect. A 603, 22 (2009). 